\title{
ORIGINAL ARTICLE \\ Spectrum of nontraumatic myelopathies in Ethiopian patients: hospital-based retrospective study
}

\author{
NJ Fidèle ${ }^{1}$ and A Amanuel ${ }^{2}$
}

Study design: This is a retrospective hospital-based study.

Objectives: The study aimed at a better understanding of the etiology, clinical presentation and treatment outcome of nontraumatic myelopathies in Ethiopian patients.

Setting: Etiologies of nontraumatic myelopathies have not been evaluated extensively in most sub-Saharan African countries. The available studies in this region were conducted before the widespread clinical use of modern neuroimaging modalities. This study was conducted in Addis Abba, Ethiopia.

Methods: We retrospectively analyzed medical files of patients with a diagnosis of myelopathy (age $\geqslant 13$ years) admitted or followed up at Tikur Anbesa Hospital between 1 January 2010 and 30 June 2013.

Results: Records of 105 patients were analyzed. The male to female ratio was 1.7. The mean age was 38.5 years. Weakness, sensory symptoms (including sensory level), back pain and sphincter dysfunction were the dominant features. Etiologies were dominated by spinal tuberculosis (23.8\%) followed by spinal cord neoplastic lesions (primary (10.5\%) and secondary neoplasms $8.6 \%$ ). Other important etiological causes were transverse myelitis (16.2\%), degenerative cervical spondylotic myelopathy (15.2\%), amyotrophic lateral sclerosis (4.8\%) and neuromyelitis optica/multiple sclerosis (3.8\%). The mortality rate was $9.5 \%$. Among the patients who died, $40 \%$ had chest infection as a complication and $70 \%$ presented with complete weakness.

Conclusion: Infections remain a major cause of spinal cord disease, and tuberculosis constitutes public health target for reducing the incidence of myelopathies. Early detection and treatment of complications may reduce the high rate of mortality and morbidity observed.

Spinal Cord (2016) 54, 604-608; doi:10.1038/sc.2015.226; published online 29 December 2015

\section{INTRODUCTION}

Myelopathies are common neurological diseases. Worldwide, the epidemiological data of nontraumatic spinal cord diseases are scarce. Some of the available studies in Sub-Saharan Africa ${ }^{1-7}$ are very old and were conducted on the basis of clinical diagnosis and spinal X-ray because of unavailability of computed tomography (CT) and magnetic resonance imaging (MRI). Spinal tuberculosis (TB) was apparently the primary cause of nontraumatic spinal cord lesions in sub-Saharan Africa and, together with neoplasms, account for almost half of the reported cases. ${ }^{1,2}$ However, with the advent of CT and MRI, the yield for positive diagnosis has greatly increased. We aimed at determining the causes of myelopathy at the Tikur Anbessa Hospital, the main teaching Hospital of Addis Ababa University, in order to improve early diagnosis and treatment strategies.

\section{PATIENTS AND METHODS}

In this retrospective study, patients with myelopathies aged $\geqslant 13$ years who presented to Tikur Anbessa Hospital from January 2010 to June 2013 were included. Medical records of patients were retrieved and a datasheet was used to collect the following data: age, sex, address, occupation, presenting symptoms and signs, investigations, treatment, duration of hospital stay and treatment outcome at hospital discharge.
Confidentiality was assured by giving each patient a unique number. The study was started after getting ethical approval from the appropriate body of the Addis Ababa University. Patients excluded from the study were patients aged $<13$ years, patients who had wrong diagnosis, patients with insufficient data for diagnosis or patients with traumatic myelopathies. The data were analyzed using SPSS 16.0 for windows (SPSS, Chicago, IL, USA).

Presumed etiologies of myelopathies were diagnosed based on collating clinical examination findings with information obtained from the neuroimaging studies, cerebrospinal fluid (CSF) and blood studies, chest X-ray findings, associated neurological and non-neurological illness/es, histology (where available) and response to treatment (for example, tuberculosis).

The diagnosis of nontraumatic myelopathy was considered in the following scenarios, used also in a similar study conducted in Yaoundé (Cameroun): ${ }^{1}$

- nontraumatic paraplegia (or paraparesis) and tetraplegia (or tetraparesis) with sensory level;

- nontraumatic paraplegia (or paraparesis) and tetraplegia (or tetraparesis) without sensory level but with spastic limbs and without cerebral functions and/or controlateral cranial nerve abnormalities;

- nontraumatic paraplegia (or paraparesis) and tetraplegia (or tetraparesis) without sensory level or spastic limbs but also without sign of peripheral nerve disease, neuromuscular junction disease and muscular disease;

- nontraumatic hemiparesis, triparesis and monoparesis with other more specific signs of myelopathy (such as up-going plantar response, bilateral

${ }^{1}$ Internal Medicine Department, Neurology unity, Butare University Teaching Hospital, Rwanda and ${ }^{2}$ Department of Neurology, Addis Ababa University, Ethiopia Correspondence: Dr NJ Fidèle, Internal Medicine Department, Neurology unity, Butare University Teaching Hospital, HUYE, Po Box 254 Butare, Rwanda.

E-mail: nshimiyimanajules@gmail.com

Received 6 June 2015; revised 15 November 2015; accepted 23 November 2015; published online 29 December 2015 
hyper reflexia) were considered as myelopathy if the brain imaging (CT or MRI) was normal with a spinal cord lesion explaining the clinical findings.

The clinical context in which we considered our presumptive diagnosis was as follows:

Transverse myelitis modified from Berman et al. ${ }^{8}$

1. acutely or subacutely developing motor, sensory and sphincter disturbance;

2. sensory level;

3. no clinical or laboratory evidence of spinal cord compression;

4. absence of other known neurological illness; and

5. lack of progression over 4 weeks.

The above-mentioned criteria were used to diagnose any inflammatory acute transverse myelitis, as it was difficult in our set-up to exclude some of the etiologies such as viral and bacterial myelitis. The infectious etiology was specified where applicable.

Neuromyelitis optica

- presence of myelitis and optic neuritis

- a contiguous spinal cord lesion on MRI extending three or more segments.

Multiple sclerosis:

- dissemination in space demonstrated on MRI by one or more T2 lesions in at least two of four multiple sclerosis-typical regions of the central nervous system (periventricular, juxtacortical, infratentorial or spinal cord);

- dissemination in time demonstrated by two clinical attacks at different times.

We considered spinal tuberculosis in the following scenarios:

- Pott's disease: if chronic myelopathy with imaging (X-ray, CT scan or MRI) suggesting Pott's disease together with or without clinical response to anti-tuberculous medication.

- intramedullar tuberculosis: intramedullar inflammatory lesion by MRI responding to anti-tuberculous medications.

- tuberculosis myeloradiculatis: subacute or chronic onset of radiculomyelitis with response to anti-tuberculous treatment.

\section{RESULTS}

Data from 105 patients who satisfied the inclusion criteria were analyzed. Sixty-six (62.9\%) were male and the mean age was 38.5 years s.d. (15; median 38 and range 14-78). A majority of our patients $(86.7 \%)$ were below the age of 55 years (Table 1$)$. The occupation was known for 35 patients, and 18 (51.4\%) among them were daily

Table 1 Age and sex distribution of 105 patients with myelopathy admitted to TAH from January 2010 to June 2013

\begin{tabular}{lccccc}
\hline \multirow{2}{*}{ Age range (years) } & \multicolumn{2}{c}{ Sex } & & Total & Percent \\
\cline { 2 - 4 } & Male & Female & & $(\%)$ \\
\hline $13-15$ & 2 & 1 & 3 & 2.9 \\
$16-30$ & 23 & 13 & 36 & 34.3 \\
$31-45$ & 20 & 14 & 34 & 32.4 \\
$46-60$ & 16 & 7 & 23 & 21.9 \\
$61-75$ & 4 & 4 & 8 & 7.6 \\
$76+$ & 1 & 0 & 1 & 1 \\
Total & 66 & 39 & 105 & \\
\hline
\end{tabular}

Abbreviation: TAH, Tikur Anbessa Hospital. laborers. Sixty- five percent of our patients were coming from out of Addis Ababa, the capital city.

The first noticed symptoms were extremity weakness in $45(46 \%)$, back or neck pain in $31(32 \%)$, numbness or paresthesia in $12(12.4 \%)$ and pain and weakness at the same time in $9(9.3 \%)$. The mean duration before consultation was 19 months, s.d. (3), whereas the median was 5 months. The mean hospital stay was 25.7 days s.d. (18.7), the range being $2-76$ days and the median being 21.5 days. The comorbidities identified with the diagnosis of myelopathy were mainly human immunodeficiency virus (HIV)/acquired immunodeficiency syndrome (nine cases) and TB (nine pulmonary and six extrapulmonary TB cases). Other recorded comorbidities included diabetes, asthma (two cases each) and tertiary syphilis, non-Hodgkin lymphoma, multinodular goiter and stage IV prostate cancer (one case each).

The clinical features are shown in Table 2. The pattern of weakness was as follows: paraplegia/paraparesis in 78 (74.3\%), quadriplegia/ quadriparesis in $17(16.2 \%)$, triplegia/triparesis in $5(4.8 \%)$, hemiparesis/hemiplegia in $3(2.9 \%)$ and monoplegia/monoparesis in $2(1.9 \%)$. Spinal MRI was performed in $78(74.3 \%)$. Spinal CT scan and spinal X-ray were performed in 4 and 23 patients, respectively.

HIV test was performed for 44 patients, and 9 (20\%) among them were positive. CD4 count was done for 8 patients $($ median $=210$, ranged from 90 to 1444). Among HIV-positive patients, 6 (66.7\%) had spinal TB as a cause of myelopathy. Others were having Burkitt lymphoma, multiple myeloma and undiagnosed myelopathy one case each. Complete cell blood count was performed for 87 patients. The hemoglobin level was $<12 \mathrm{~g} \mathrm{dl}^{-1}$ for 13 (15\%). Erythrocyte sedimentation rate was determined in $43(41 \%)$ patients, and it was above $20 \mathrm{~mm} \mathrm{hr}-1$ in 30 (69.8\%). Among patients with inflammatory and neoplastic causes, erythrocyte sedimentation rate was abnormal for $90 \%$ and $85.7 \%$, respectively. The etiologies of myelopathy are depicted in Table 3. The causes were infectious in $28(26.7 \%)$, degenerative in 24 (22.9\%), malignancy in 20 (19\%), inflammatory in $19(18 \%)$, toxic metabolic in $1(0.9 \%)$ and for $13(12.4 \%)$ patients the cause was not known.

The infectious causes included spinal TB in 25 patients $(23.8 \%$ of all causes of myelopathy; Figure 1). The degenerative causes of myelopathy were dominated by degenerative cervical spondylotic myelopathy, in 16 patients (15.2\% of all myelopathy). The mean age in this etiological group was 44.1 years, with the minimum being 30 years and maximum being 70 years.

Eight-nine patients received medical treatment. Steroids (predinisolone or dexamethasone) were given to $45(50.5 \%)$ of our

Table 2 Clinical features of 105 patients with myelopathy at TAH

\begin{tabular}{lcc}
\hline Clinical feature & Frequency & Percentage \\
\hline Weakness of extremities & 105 & 100 \\
Pain (back, neck and radicular) & 73 & 69.5 \\
Sphincter dysfunction & 60 & 57 \\
Sensory level & 48 & 45.7 \\
Paralysis(complete weakness) & 33 & 31.4 \\
Fever & 18 & 17 \\
Back tenderness & 12 & 11.4 \\
Vertebral deformity & 9 & 8.5 \\
Sweat level & 8 & 7.6 \\
Respiratory failure & 3 & 2.8 \\
Total & 105 & \\
\hline
\end{tabular}

Abbreviation: TAH, Tikur Anbessa Hospital.

NB: Please note that a patient could have more than one feature. 
patients. The dose range was $5-60 \mathrm{mg}$ per day for prednisolone and $4 \mathrm{mg}$ bid- $8 \mathrm{mg}$ quid for dexamethasone. Patients with transverse myelitis, spinal $\mathrm{TB}$ and neoplasm received this therapy in a big proportion $(68.75 \%, 59.01 \%$ and $82 \%$, respectively). All patients with neuromyelitis optica and multiple sclerosis were treated with steroids. All patients with amyotrophic lateral sclerosis received amitriptyline. Anti-TB was given to $23(25.5 \%)$ patients, and nonsteroidal antiinflammatory drugs were used for $15(16.7 \%)$ patients among 90 who received medical treatment.

In total, $22(21 \%)$ of our participants had surgical intervention and these were having degenerative cervical spondylotic myelopathy, neoplasms and spinal TB (10, 6 and 4 patients, respectively) as cause of their spinal cord diseases. Drainage was performed for two patients diagnosed with syrinx.

The types of operations performed were decompressive laminectomy for degenerative cervical spondylotic myelopathy with improvement in 5 (50\%), tumor removal and abscess drainage for TB spondylitis. Improvement was noted for all operated patients with TB spondylitis. Among the four operated patients with primary

Table 3 Causes of myelopathy in 105 patients admitted to TAH from January 2010 to June 2013

\begin{tabular}{lcc}
\hline Cause & Frequency & Percent \\
\hline Spinal Tuberculosis & 25 & 23.8 \\
Transverse myelitis & 17 & 16.2 \\
DCSM & 16 & 15.2 \\
Primary Neoplasm & 11 & 10.5 \\
Spinal metastasis & 9 & 8.6 \\
ALS & 5 & 4.8 \\
NMO/MS & 4 & 3.8 \\
others $^{\text {a }}$ & 5 & 4.8 \\
Undiagnosed $^{\mathrm{b}}$ & 13 & 12.4 \\
Total & 105 &
\end{tabular}

Abbreviations: ALS, amyotrophic lateral sclerosis; DCSM, degenerative cervical spondylotic myelopathy; MS, multiple sclerosis; NMO, neuromyelitis optica; TAH, Tikur Anbessa Hospital. aNB others: syrinx $=2$, neurolathrysim $=1$, compression due to syphilitic aneurysm $=1$, hereditary spastic paraparesis $=1$.

bAll the undiagnosed patients had chronic progressisve spastic paraparesis/paraplegia. neoplasm, three have improved and one patient who had ependymoma with syrinx remained in the same clinical conditions. Among the three patients who were operated for spinal metastasis, one remained in the same condition and the other two died.

In general, $70(66.7 \%)$ of our patients had no improvement (53 remained in the same clinical conditions, 4 deteriorated from the baseline but without death and 10 patients died), whereas 35 $(33.3 \%)$ had at least partial improvement. The common presentation for those patients who died was complete paralysis (7 among 10 deaths), whereas the common complication was chest infection (4 among 8 deaths). Seventy-one percent of admitted patients with spinal TB had improvement. Seven patients with transverse myelitis did not have any change in terms of improvement, whereas 3 died in the hospital. The remaining 3 had some degree of improvement. Complications were identified among $24(22.9 \%)$ patients, and these include urinary tract infection in $11(10.5 \%)$, chest infection in $9(8.6 \%)$, bed sores in $4(3.8 \%)$, muscle contractures in $1(0.9 \%)$, deep venous thrombosis in $1(0.9 \%)$ and pulmonary embolism in $1(0.9 \%)$.

The mortality rate was $9.5 \%$. Immediate causes of death were sepsis (five cases; 50\%), respiratory failure (two cases), hypokalemia, pulmonary thromboembolism and postoperative bleeding with hypovolemic shock (one case each).

\section{DISCUSSION}

This study of nontraumatic spinal cord diseases at the Tikur Anbessa Hospital is among the very few dedicated to myelopathies in subSaharan Africa particularly in the era of MRI and CT imaging modalities. The mean age of 40 years is similar to that reported in other African series of 30-40 years. ${ }^{1,2,4}$ This may reflect the relatively young population found in Ethiopia, with $99.3 \%$ of the population under 55 years. ${ }^{9}$ The gender bias in utilization of health facilities was pointed out in Addis Ababa previously by Reniers et al. ${ }^{10}$ in 2005 and could be at least partly the reason for the male predominance found in our study (male/female ratio: 1.7). However, the number of men in the above-mentioned studies ${ }^{1,2,4}$ outnumbered the number of women as well (male/female ratio: from 1.3 to 3.1). The clinical presentation of our patients did not differ from previous reports of spinal cord diseases from other developing countries, with weakness, sensory
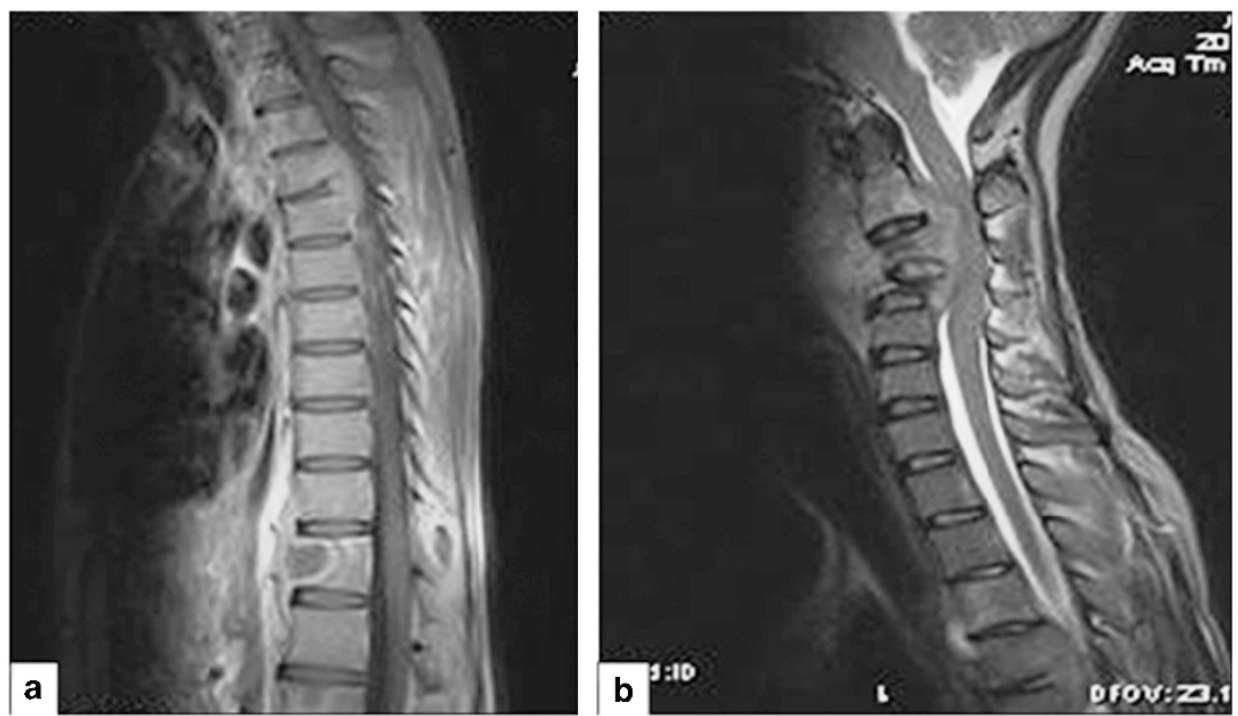

Figure 1 Cervical and thoracic TB spondylitis T1- and T2-weighted MR images (a and $\mathbf{b}$, respectively) showing disc destruction, wedge-shaped vertebral fracture, paravertebral abscess, involving the cervical and thoracic spine with spinal cord compression. These findings are suggestive of TB spondylitis in a 35-year-old female patient who presented with progressive weakness of the upper and lower extremities of 3 months duration. 
symptoms (including sensory level), back pain and sphincter dysfunction being the dominant features. ${ }^{1,11}$

Delay before neurological consultation has been also reported long in Tanzania where the mean duration before consultation was 8 weeks. ${ }^{12}$ The inclusion of outpatients in this study could falsely exaggerate the time before consultation in our study where the mean was 18.9 months. In fact, some of the patients could present as outpatients to our tertiary care center after several months of followup in different primary and secondary care centers. The number of neurologists was 15 (personal communication), all practicing in the capital city at the time of the study. This, together with the lack of modern diagnostic modalities, could contribute to the delay of diagnosis and referral. The damage that occurs to the spinal cord is in many cases irreversible. The delay to diagnosis has clearly contributed to the worse outcome in many patients $(66.7 \%$ without any improvement). Timely identification of the cause of myelopathy can reduce disabilities, mortality and social burden from late management of these conditions. HIV test was not systematic in our study. However, the frequency of TB $(22.9 \%)$ and HIV $(8.5 \%)$ as comorbidities and predisposing factors to spinal cord affection in our study is in accordance with prevalence of these conditions in Ethiopia. ${ }^{13}$ The incidence of TB in Ethiopia in 2007 was estimated at 152 and 341 per 100000 populations for new smear-positive pulmonary and all forms of TB, respectively. ${ }^{13}$

The prevalence of HIV among the urban and rural populations in 2008 was estimated at $7.7 \%$ and $0.9 \%$, respectively. ${ }^{13}$ Despite the lack of some more important paraclinical tests to reach the definitive diagnosis, the introduction of MRI in Ethiopia (74.3\% had spinal MRI) may have reduced the number of patients with no definitive diagnosis (12.4\%), although in centers equipped with MRI this figure is still up to $16.5 \%$ of patients with acute myelopathy. ${ }^{1}$ The frequency of anemia $(16.1 \%)$ in the present study is in accordance with the endemicity of anemia in the country. The Ethiopia Demographic and Health Survey 2011 report showed that $17 \%$ of women aged 15-49 years are anemic and $11 \%$ of men aged $15-49$ years are anemic. ${ }^{9}$ The sensitivity of erythrocyte sedimentation rate $(85.7 \%$ for neoplastic and $90 \%$ for inflammatory) to screen myelopathies for neoplastic and infectious conditions found in the present study was described earlier by Deyo RA et al. ${ }^{14}$ in 1988 (76-95\% infection and 78\% malignancy).

In the present study, we observed a relatively high frequency of transverse myelitis (16\%) compared with other African series (from 0 to $13.1 \%) .{ }^{1-7}$ In the previous study in Ethiopia ${ }^{4}$ mentioned above, this entity was attributed to HIV and was lumped together with vacuolar myelopathy to be called myelitis because of HIV (17\%).

Even though HIV test was not done routinely for all patients in our study, over half $(64 \%)$ of the patients with transverse myelitis were tested and all were negative, implying a relatively low frequency of HIV myelitis compared with the previous study in the same hospital. However, among 13 paraparetic patients with no diagnosis, 9 did not have HIV test, therefore there was difficulty of excluding HIVassociated vacuolar myelopathy. The lack of complete analysis of CSF including anti-aquaporin four antibodies and oligoclonal bands precluded a proper final diagnosis of myelitis. The postinfectious, idiopathic and myelitis secondary to systemic inflammation were lumped under the denominator transverse myelitis. Myelopathies due to HTLV-1 could not be identified because of the absence of serological tests. Apart from the above exceptions, other etiologies are almost similar to that reported from other African countries, with tumors and tuberculosis accounting for close to half of the cases. ${ }^{1,4,5,7}$

The recovery rate for the spinal $\mathrm{TB}$ was $71.4 \%$, with partial recovery. A recovery rate with anti-TB alone was previously reported by Njoku et al. ${ }^{15}$ in 2007 in Nigeria, with $25 \%$ full recovery, $9.8 \%$ partial recovery and no recovery in $10.86 \%$. In all, $50 \%$ of their patients left the study before conclusion, and there was no recovery status documented. Antibiotics combined with surgery offer the functional recovery as high as $90 \%{ }^{16}$

The poor outcome observed in the present study for patients with transverse myelitis (same clinical condition for 64.7\%) can be attributed partly to the delay before administration of corticosteroids. Inadequate management of complications associated with bedridden patients also account for the deaths in this group of etiologies. This is in contrast to the usually reported prognosis of transverse myelitis regarding the clinical evolution after nadir. Generally, the patients are roughly divided into three groups: one-third will recover completely; one-third of patients present residual symptoms; and the remaining have no improvement at all. ${ }^{17}$

Almost all patients with spinal metastasis had poor outcome at discharge: they either died (three patients) or remained in the same clinical conditions (five patients). The management also was not adequate because of inaccessibility to radiotherapy and above all the failure to determine the primaries in order to select the appropriate chemotherapy. Peter W et al. ${ }^{18}$ in 2002 evaluated the pattern of complications in nontraumatic myelopathy for patients admitted in neurorehabilitation program. Even though we cannot compare his findings with the current study, which included outpatients and evaluated only complications at discharge, the UTI remained the predominant complication ( 32.8 versus $10.5 \%$ in our study). It is believed that autonomic hyperreflexia is less prevalent in patients with incomplete SCI lesion. ${ }^{19}$ Most of the nontraumatic spinal cord diseases in our population were incomplete, and this may partially account for our lower prevalence of this complication.

The restrospective nature of the study compromised adequate data collection. Some patients' files were incomplete, and we lacked information on many items such as profession, description of imaging finding, HIV test, past medical history and so on. Specific etiologic diagnosis was sometimes impossible because of limited availability of laboratory tests including proper CSF analysis and lack of facilities for MRI imaging.

In conclusion, the etiologies of nontraumatic myelopathies in our study did not differ from other sub-Saharan series, with spinal tuberculosis being the leading cause $(23.6 \%)$ and together with neoplasms constitute $\sim 40 \%$ of cases. The accessibility to MRI was satisfactory for our patient $(74.5 \%)$, and it has reduced the number of patients without diagnosis. According to our findings, spinal X-ray can help achieve the diagnosis alone in $45 \%$ of patients with spinal TB but rarely in other cause of compressive myelopathy. Chance to survive is worse for those patients who developed complications, especially chest infection (probably because of subsequent respiratory failure and lack of adequate respiratory support) and those with complete paralysis because of the increased probability to develop complications.

Infections remain a major cause of spinal cord disease and TB constitute public health target for reducing the incidence of myelopathies. A prospective study with proper CSF analysis designed for the determination of etiologic causes of transverse myelitis is needed to clarify the importance of this group of spinal cord diseases in our set-up. Early detection and treatment of myelopathy and its complications may reduce the high rate of mortality and morbidity observed in our study.

\section{DATA ARCHIVING}

There were no data to deposit. 


\section{CONFLICT OF INTEREST}

The authors declare no conflict of interest.

1 Lekoubou Looti AZ, Kengne AP, Djientcheu Vde P, Kuate CT, Njamnshi AK. Patterns of non-traumatic myelopathies in Yaoundé (Cameroon): a hospital based study. J Neurol Neurosurg Psychiatry 2010; 81: 768-770.

2 Draulans N, Kiekens C, Roels E, Peers K. Etiology of spinal cord injuries in Sub-Saharan Africa. Spinal Cord 2011; 49: 1148-1154.

3 Van den Berg MEL, Castellote JM, Mahillo-Fernandez I, De Pedro-Cuesta J. Incidence of spinal cord injury worldwide: a systematic review. Neuroepidemiology 2010; 34: 184-192.

4 Zenebe G. Myelopathies in Ethiopia. East Afr J Med 1995; 72: 42-45.

5 Nyame PK. An aetiological survey of paraplegia in Accra. East Afr J Med 1994; 71: 527-530.

6 Ogunniyi A, Shokunbi MT, Oluwole OSA, Adeyinka A, Malomo A, Adebiyi AA. Non-traumatic spinal cord diseases in Ibadan, Nigeria: aetiology and prognostic factors. Central Afr J Med 1995; 41: 50-54.

7 Parry O, Bhebe E, Levy LF. Non-traumatic paraplegia in a Zimbabwean population - a retrospective study. Central Afr J Med 1999; 45: 114-118.

8 Berman M, Feldman S, Alter M, Zilber N, Kahana E. Acute transverse myelitis, incidence and etiological considerations. Neurology 1981; 31: 966-971.
9 Central Statistical Agency (CSA) (Ethiopia) and ORC Macro Ethiopia Demographic and Health Survey 2011. Addis Ababa, Ethiopia and Calverton, Maryland, USA.

10 Reniers G, Araya T, Schaap A, Kumie A, Kebede D, Nagelkerke N et al. Monitoring cause-specific adult mortality in developing countries: A comparison of data sources for Addis Ababa and its implications for policy and research. Soc Sci Med 2005; 61 : 1952-1957.

11 Modi G, Ranchhod J, Hari K, Mochan A, Modi M. Non-traumatic myelopathy at the Chris Hani Baragwanath Hospital, South Africa: the influence of HIV. Q J Med 2011; 104: 697-703.

12 Scrimgeour EM. Non-traumatic paraplegia in northern Tanzania. Br Med J 1981; 283. 975-978.

13 Federal Ministry of Health of Ethiopia (2007) Implementation Guideline for TB/HIV Collaborative Activities in Ethiopia. Addis Ababa, Ethiopia.

14 Deyo RA, Diehl AK. Cancer as a cause of back pain: frequency, clinical presentation, and diagnostic strategies. J Gen Intern Med 1988; 3: 230-238.

15 Njoku CH, Makusidi MA, Ezunu EO. Experiences in management of Pott's paraplegia and paraparesis in medical wards of Usmanu Danfodiyo university teaching hospital, Sokoto, Nigeria. Ann Afr Med 2007; 6: 22-25.

16 Joseph R, Arman S. Infectious myelopathies. Semin neurol 2002; 22: 133-142.

17 Sá MJ. Acute transverse myelitis: A practical reappraisal. Autoimmun Rev 2009; 9: 128-131.

18 New PW, Rawicki HB, Bailey MJ. Non-traumatic spinal cord injury: demographic characteristics and complications. Arch Phys Med Rehabil 2002; 83: 996-1001.

19 Kewalramani LS. Autonomic dysreflexia in traumatic myelopathy. Am J Phys Med 1980; 59: $1-21$. 